\title{
The Health Efficacy of Aloe and
}

\section{Its Development and Utilization}

\author{
Yan Li \\ Biology Department, Dezhou University \\ Dezhou 253023, China \\ E-mail: lylxy0524@126.com
}

\begin{abstract}
With the advance and development of the modern technology, more new chemical constituents of aloe have been found. It has become hot to study the pharmacological effects and application value of aloe. This paper concentrates on the varieties of aloe, the main chemical constituents and the health efficacy. And the future development and utilization of aloe products are also discussed.
\end{abstract}

Keywords: Aloe, Chemical composition, Nutritional value

Aloe belongs to the aloe family of liliaceous species. It is of evergreen perennial herbs and lives mainly in tropical and subtropical zones. Aloe is juicy and rich in polysaccharides, aloin, a variety of essential amino acids, vitamins, minerals and other active ingredients, with the efficacy of medical treatment, cosmetic repair and health care. As it is editable and ornamental, it gets many nicknames of "good medicine with many efficacies", "natural beautician", "source of life energy" and "a family doctor", etc. (Li, 2000, pp19-23). The ratio of the nutrition is in line with the standard of the UN Food and Agriculture Organization thus it is known as the "best health food of the $21^{\text {st }}$ century". In recent years, considerable progress has been made in pharmacological research on aloe and there has appeared in the market some aloe food, medicine, health care products and cosmetics, which seems very popular.

\section{Aloe species}

There are at least more than 300 Aloe species, of which 250 or so are in the African continent; About 40 are in Madagascar and other 10 in Arab and other places. But only six species are edible, and those with medical value are mainly Aloe Vera and Aloe Saponaria.

\subsection{Aloe Saponaria}

The origin of Aloe Saponaria is in Africa, mainly in the northeastern part of Cape Island and Transvaal state. In addition, it is also planted in Hawaii, Florida of the United States. And in Japan it is found to be cultivated, too. This kind of aloe has a short stem with green leaves, spotted with white stripes, of clear texture about $50 \mathrm{~cm}$ long. Its rhizome elongation spreads fast, germinates and grows up in groups. Big, thick and fleshy leaves contain much gelling with mild resistance. It is also of a higher ornamental value.

\subsection{Aloe Chinensis}

Aloe Chinensis is mainly in Hainan, Guangdong, Fujian, Yunnan and some cities of Taiwan Province. It likes to live among the bushes in hillside and sandy beaches. As a variant of aloe, it is similar with West Indian aloe in appearance. There are white spots in its basal fleshy leaves and stems with a mighty meristematic ability and strong adaptability. Aloe Chinensis has a certain value of cosmic repair and medicine.

\subsection{Aloe Vera}

Aloe Vera, also named as La Fan aloe Vera, or Real aloe Vera, is widely used in food, medicine and cosmetic products. Aloe Vera, originated in northern Africa, is now cultivated more in America. And in Japan, South Korea, Taiwan province and Hainan Island of China, large amount of Aloe Vera is cultivated for commercial purpose of extracting aloe juice. 


\subsection{Aloe Arborescens}

Aloe Arborescens, with small and thin leaves, is unique to Japan. It is widely planted and employed as a Japanese folk medicine and health food for oral treatment and exterior use against constipation, indigestion, etc. It can also be made for a variety of food, beverages, cosmetic products and so on.

\section{Chemical constituents of aloe}

The aloe has extremely complicated chemical composition, more effective than other plants. It has been proved that aloe has more than 160 chemical compositions, among which 72 are functional, such as anthraquinone compounds, (Kong, 2003, pp.71-75) polysaccharides, glucoside, lipid, organic acids, enzymes, amino acids, antibiotics, vitamins, etc. In addition, water in a fresh aloe leaf accounts for $96.0 \%$ to $96.5 \%$ of the whole weight of the leaf. And this kind of biological water is completely natural, known as smooth water.

\subsection{Anthraquinone compounds}

Anthraquinone compounds in aloe have been considered as the main organic and active anti-inflammatory ingredient. Of them, 20 such as aloe-emodin and barbaloin are the most important. Emodin is one of the most basic components. These substances are the most active in living bodies.

Aloin has a very good effect by making the intestinal bacteria decomposed and activated, promote the contraction of intestinal muscles, speed up bowel movements. Barbaloin can inhibit the free histamine, and it has good effect on asthma, allergic rhinitis, pollen allergies, etc.

\subsection{Polysaccharides}

Polysaccharide is a kind of macromolecular compound with different physiological functions, containing glucose, fructose, mannose, sucrose, galactose, xylose, arabinose, rhamnose, glucuronic acid and other components. These polysaccharides are the major components of mucus, which have effective functions in enhancing the immune system of human bodies and preventing aging and treating chronic allergy.(Hu, 2003. pp. 158-161). Aloe polysaccharide, mannan in particular, can significantly release the symptoms of cancers and AIDS, reduce the infections, and promote the regeneration of burns due to radiation.

\subsection{Amino acid and organic acid}

Aloe leaves contain 19 amino acids such as glutamic acid, and threonine, etc. Among them, 8 such as arginine are essential. These amino acids can be beneficial to skin metabolism, prevent ultraviolet from hurting and oxidizing the skin, and promote the regeneration of the skin cells, thus increasing their abilities against radiation. The roots, stems, and leaves of aloe contain acetate and other organic acids, which can involve in biochemical processes in the human body, directly regulate $\mathrm{pH}$ degrees in the stomach, as is conducive to the prevention of diseases. They can also make the metabolism of sugar and protein normal.

\subsection{Vitamin and mineral elements}

The juice of aloe leaves contains a variety of vitamins and minerals, which can promote the metabolism of protein, sugar and fat, regulate the physiological functions, and maintain the osmotic pressure and acid-base balance. Vitamins in aloe are conductive to the skin care and can enhance immune functions of body fluid. They can be helpful to the anti-cancer drugs to enhance the ability against bacteria and viral tumor or kill dangerous cells.

Germanium in aloe exists in an organic form. Organic germanium has a good effect on treating uterine and lung cancer, and cerebral thrombosis and arteriosclerosis as well. Tens of thousands of patients in the world have turned to the application of organic germanium, and the seriousness of their diseases has been lessened. Therefore, organic germanium in aloe is known as the Life Germanium in the 21 st century.

\subsection{Active enzymes and the others}

Aloe juice contains a variety of active enzymes. It can decompose the dead cells of the surface skin and play a certain role in metabolism of skin, thus producing a good effect on skincare by improving the smoothness of skin and nourishing the hair. SOD has efficacy of anti-aging, keeping young, treating rheumatoid arthritis and rheumatoid arthritis. Phytoagglutinin is conductive to the growth of anti-infectious lymphocytes. It helps to enhance the function of the lymphocytes and improve the immune function in the human body to raise the ability against various diseases. Aloe contains interleukin too, which can promote the recovery of immune function to increase resistant ability against many diseases.

There is in the fresh leaves of aloe an important element of "water", which is quite different from the ordinary natural water. It flows along the vessels at a very fast speed, twice as fast as the ordinary water. The juice of aloe contains a variety of similar water-soluble polymer materials. The "water" can make the effective ingredient of mucopolysaccharide in aloe leaves rapidly absorbed by human body to help speed up the cell division and promote cell metabolism. Therefore, according to the pharmacists, it is perhaps one of the important reasons that aloe can treat many 
diseases.

\section{The development and utilization of aloe}

Aloe, as one of the green resources of high economic and medicinal value, has been explored and used widely increasingly. Developing and utilizing its economic value has attracted more attention from the countries and their governments where aloe is planted. Now aloe is mainly used in the following five aspects.

\subsection{Health products}

Aloe is nutritious with the functions of strengthening stomach, relaxing the bowels, and softening vascular. It is known as the best health product of the 21 st century. At present, aloe has been widely used as a raw material or an additive in health products. Aloe can be made into health wine or tea. As for the health products for external use, health cream or dew can be made from aloes, too. Aloe health products can be used externally or internally to keep gastrointestinal, cardiovascular, or skin health. There is now Aloe Capsule at the market manufactured by Guangzhou Yipintang biological detoxification Co., Ltd., and Aloe Arborescens Tablets by Fujian Changle Jianyou Aloe Products Co., Ltd.

\subsection{Cosmetic products}

In the course of the development of aloe industry, aloe cosmetics grow fastest and are easiest to be accepted by consumers. In 1980s, when a selecting activity named Best Effective Elements of Cosmetic was held in the United States, aloe won the second to vitamin as the cosmetic additive. Aloe cosmetics can be divided into 4 major categories, namely, cleaning and nursing, skin care, skin beautifying, and other products with special features. Aloe in skin cream has the properties of suppressing the pimples, nourishing and keeping moisture, removing the dead skin and resisting aging. Aloe powder in the shower products and soaps has an excellent anti-irritant and deodorant effect. It also can apparently enrich the lipid and moistening the skin.

\subsection{Medicine}

Aloe is a medicinal plant of high value, known as the "medicinal plant kit". Aloe can be used as a raw material to produce medicinal capsule, injection, tablets and other medicines, which can be applied in medical, surgical, gynecologic, pediatrics, etc. Aloe has good efficacy for treating hemorrhagic diseases, burns, skin disorders and so on. Especially in the treatment of burns, flailing injuries, incised wound, it is a specific drug in promoting the wound organs regenerate and greatly relieving the pain. In addition, a German scientific research institute also found that aloe can not only heal the external wounds but also can heal a variety of internal gastro-intestinal ulcers, promote digestion and absorption of nutrients. Therefore, it is promising to further develop aloe pharmaceutical products.

\subsection{Food}

Aloe belongs to a health food. Its nutrition and safety is in line with the requirements of the UNFAO. The edible aloes have all kinds of physical nutrition necessary to the people. Aloe, as a raw material for medicine and food, can be consumed as a vegetable and processed into food or beverages. Moreover, these foods are not only nutritious, but also have their own unique flavor.

\subsection{Ornamental value}

Aloe is also of great ornamental value. Furthermore, it is evergreen and much easier to plant. There are many species such as aloe of green leaves or mottling, or rosette. Aloes can be planted in pots or just in ground to beautify the surroundings. Aloe releases oxygen during the daylight and can absorb carbon dioxide at night to purify the air indoors. Aloe, as a natural air monitor, can refresh the air and drive off the mosquitoes and other insects. It also can effectively absorb CO2, SO2 and other harmful gases. (HU, 2001, pp. 12-15). When the harmful gases in air arrive at a certain limit and aloes absorb these gases, their leaves will display some brown or black spots. The sign can remind people of the air pollution.

\section{Future applications of Aloes}

With the advance of science and technology and the continuous improvement of people's living standard, human beings long for health and longevity and hope to be closer to nature and using all-natural medicine, food and cosmetics. And the various functions of aloe can meet the new requirements of the human race. At present, in the structure of aloe products, the market of aloe cosmetics has been developed rapidly. With the people's growing understanding for health theory of homology of medicine and food, some research institutes have deeply explored the quality of identification and aloe nutrition. Since a large number of financial, material and human resources have already been invested in studying aloe's functions of regulating enterogastric, releasing the alcohol detoxification, lowering the blood pressure and alleviating diabetes and chronic cardiovascular diseases, regulating the immune functions, I believe that in the coming decade, aloe products in China will become the daily food and medicine that ordinary people can afford to consume. Aloe health products will also show its strong momentum of development. 


\section{References}

Hu, Yun. (2003). New progresses in biological active composition and functions of aloes. Food Science.

Hu, ZhengHai, Shen, Zonggen and Li, Jingyuan. (2001). Relationship of Aloe leaf structure and anthraquinones. Chinese Herbal Medicine.

Kong, Xiangrong and Wang, Zongwei. (2003). Polysaccharide chemical structure and biological activity of aloe. Herbal Medicine Abroad.

Li, Yunzheng, Qin, Haiyuan and Wang, Qinghua. (2000). Progress of applied research of aloe at home and abroad. Progress of Chemical Engineering. 